\title{
COINCIDENCE THEOREM AND SADDLE POINT THEOREM
}

\author{
H. KOMIYA
}

\begin{abstract}
We discuss Browder's coincidence theorem and derive a saddle point theorem from it.
\end{abstract}

We always assume the Hausdorff separation axiom in topological structures. Let $X$ be a topological space and let $Y$ be a nonempty subset of a linear topological space $F$. By a multi-valued mapping $A$ of $X$ into $Y$, we mean that to each point $x$ of $X, A$ assigns a subset $A(x)$ of $Y$. A multi-valued mapping $A$ is said to be convex-valued (resp. closed convex-valued) if $A(x)$ is nonempty and convex (resp. nonempty, closed and convex) for each $x$ in $X$. A multi-valued mapping $A$ is said to be upper semicontinuous if for each point $x$ of $X$ and each zero-neighborhood $V$ of $F$, there exists a neighborhood $U$ of $x$ such that $A(u) \subset A(x)+V$ for all $u$ in $U$. The upper semicontinuity of $A$ is equivalent to the closedness of the graph $\operatorname{Gr}(A)=\{(x, y) \in X \times Y: y \in A(x)\}$ of $A$ in $X \times Y$, if $A$ is closed-valued and $Y$ is compact.

There exist two fundamental fixed point theorems for multi-valued mappings. One is Kakutani-Fan's fixed point theorem:

Let $X$ be a nonempty compact convex subset of a locally convex linear topological space. Let $A$ be an upper semicontinuous and closed convex-valued mapping of $X$ into $X$. Then $A$ has a fixed point, that is, a point $x_{0}$ of $X$ such that $x_{0} \in A\left(x_{0}\right)$.

The other is Fan-Browder's fixed point theorem:

Let $X$ be a nonempty compact convex subset of a linear topological space. Let $B$ be a convex-valued mapping of $X$ into $X$ such that $B^{-1}(y)=\{x \in X: y \in B x\}$ is open in $X$ for each $y$ in $Y$. Then $B$ has a fixed point.

Kakutani and Fan's theorem has been established by Kakutani [8] in case $X$ is contained in a finite dimensional space, and Fan [4] has generalized it to the present form. Fan and Browder's theorem first appeared in [5] implicitly, and the present form is found in [1].

Browder [2] has combined the two fixed point theorems and obtained a coincidence theorem [2, Theorem 3]. We point out that Browder's coincidence theorem is implicitly contained in $\mathrm{Ha}$ [7, Theorem 3] and obtain a generalization of the coincidence theorem with a simple proof using Ha's lemma.

THEOREM 1. Let $X$ be a nonempty convex subset of a linear topological space $E$ and let $Y$ be a nonempty compact convex subset of a linear topological space $F$. Let $A$ be an upper semicontinuous and closed convex-valued mapping of $X$ into $Y$.

Received by the editors December 11, 1984.

1980 Mathematics Subject Classification. Primary 47H10, 49A40.

Key words and phrases. Coincidence theorem, saddle point, fixed point.

(C) 1986 American Mathematical Society $0002-9939 / 86 \$ 1.00+\$ .25$ per page 
Let $B$ be a convex-valued mapping of $Y$ into $X$ such that $B^{-1}(x)$ is open in $Y$ for each $x$ in $X$. Then there exist a point $x_{0}$ of $X$ and a point $y_{0}$ of $Y$ such that $y_{0} \in A\left(x_{0}\right)$ and $x_{0} \in B\left(y_{0}\right)$.

Proof. By [2, Proposition 1] there exists a continuous mapping $p$ of $Y$ into the convex hull $S$ of a finite number of points of $X$ such that $p(y) \in B(y)$ for each $y$ in $Y$. Then there exists a point $x_{0}$ of $S$ such that $x_{0} \in p\left(A\left(x_{0}\right)\right)$ by [7, Lemma $2]$, and hence there exists a point $y_{0}$ of $A\left(x_{0}\right)$ such that $x_{0}=p\left(y_{0}\right) \in B\left(y_{0}\right)$.

It is easily seen that Fan and Browder's theorem is derived from Theorem 1 by setting $X=Y$ with $E=F$ and $A$ the identity mapping of $X$. Moreover we can easily derive Kakutani and Fan's theorem from Theorem 1:

Let $X=Y$ with $E=F$ and $B_{U}(y)=\{x \in X:(x, y) \in \Delta+U \times U\}$ for each $y$ in $X$ and each open convex zero-neighborhood $U$ of $E$, where $\Delta$ is the diagonal $\{(x, x): x \in X\}$ of $X$. Then there exists a net $\left\{\left(x_{U}, y_{U}\right)\right\}$ directed by the system of open convex zero-neighborhoods of $E$ such that $\left(x_{U}, y_{U}\right)$ belongs to the set $\operatorname{Gr}(A) \cap \operatorname{Gr}\left(B_{U}\right)$ for each $U$. Since $\operatorname{Gr}(A)$ is compact, there exist a point $\left(x_{0}, y_{0}\right)$ of $\operatorname{Gr}(A)$ and a subnet of $\left\{\left(x_{U}, y_{U}\right)\right\}$ converging to $\left(x_{0}, y_{0}\right)$. Then $x_{0}$ must be equal to $y_{0}$ by the definition of $B_{U}$, and hence we have $x_{0} \in A\left(x_{0}\right)$.

We can also derive from Theorem 1 the following theorem due to Simons [10] which has generalized and unified fixed point theorems for multi-valued mappings due to Browder [2], Fan [6], and Takahashi $[\mathbf{1 1}, \mathbf{1 2}]$.

THEOREM 2 [10, THEOREM 4.5]. Let $X$ be a nonempty compact convex subset of a linear topological space $E$ and let $B$ be a convex-valued mapping of $X$ into the topological dual space $E^{\prime}$ of $E$ such that $B^{-1}\left(x^{\prime}\right)$ is open in $X$ for each $x^{\prime}$ in $E^{\prime}$. Then there exist a point $x_{0}$ of $X$ and a point $x_{0}^{\prime}$ of $E^{\prime}$ such that $x_{0}^{\prime} \in B\left(x_{0}\right)$ and $\left\langle x_{0}, x_{0}^{\prime}\right\rangle=\max _{x \in X}\left\langle x, x_{0}^{\prime}\right\rangle$.

PROOF. Let $A\left(x^{\prime}\right)=\left\{x \in X:\left\langle x, x^{\prime}\right\rangle=\max _{x \in X}\left\langle x, x^{\prime}\right\rangle\right\}$. We endow $E^{\prime}$ with the strong topology, that is, the uniform convergence topology on the bounded sets of $E$. It is easily seen that $A$ is convex-valued. Let $\left\{\left(x_{\alpha}, x_{\alpha}^{\prime}\right)\right\}$ be a net in $\operatorname{Gr}(A)$ converging to a point $\left(x, x^{\prime}\right)$ of $X \times E^{\prime}$. Since the net $\left\{x_{\alpha}^{\prime}\right\}$ converges to $x^{\prime}$ uniformly on $X$, the net $\left\{\left\langle x_{\alpha}, x_{\alpha}^{\prime}\right\rangle\right\}$ converges to $\left\langle x, x^{\prime}\right\rangle$. Hence for any point $u$ of $X$, we have the inequalities

$$
\left\langle x, x^{\prime}\right\rangle=\lim _{\alpha}\left\langle x_{\alpha}, x_{\alpha}^{\prime}\right\rangle \geq \lim _{\alpha}\left\langle u, x_{\alpha}^{\prime}\right\rangle=\left\langle u, x^{\prime}\right\rangle .
$$

Hence $\left(x, x^{\prime}\right)$ belongs to $\operatorname{Gr}(A)$ and $\operatorname{Gr}(A)$ is closed. Therefore $A$ is closed convexvalued and upper semicontinuous. Then we can apply Theorem 1 to the multivalued mappings $A$ and $B$ and obtain the desired result.

We finish our discussion with a saddle point theorem which can be derived from Theorem 1. A real-valued function $f$ on a convex set $X$ is said to be quasi-convex if the set $\{x \in X: f(x) \leq r\}$ is convex for each real number $r$. If $-f$ is quasiconvex, then $f$ is said to be quasi-concave. Let $\left\{f_{\nu}: \nu \in I\right\}$ be a family of realvalued functions on a topological space $X$. The family $\left\{f_{\nu}: \nu \in I\right\}$ is said to be equicontinuous if for any point $x$ of $X$ and any positive number $\delta$, there exists a neighborhood $U$ of $x$ such that $\left|f_{\nu}(u)-f_{\nu}(x)\right|<\delta$ for all $u$ in $U$ and $\nu$ in $I$. We denote by $C(X)$ the Banach space of all real-valued bounded continuous functions with the uniform norm. 
THEOREM 3. Let $X$ be a nonempty convex subset of a linear topological space and let $Y$ be a nonempty compact convex subset of a linear topological space. Let $f$ be a real-valued continuous function on the product space $X \times Y$ of $X$ and $Y$ which is quasi-concave in its first variable and quasi-convex in its second variable and satisfies $\sup _{x \in X} \min _{y \in Y} f(x, y)<+\infty$. Let the family $\{f(x, \cdot): x \in X\}$ of realvalued functions on $Y$ be equicontinuous and closed in the Banach space $C(Y)$. Then $f$ has a saddle point $\left(x_{0}, y_{0}\right)$, that is, $\left(x_{0}, y_{0}\right)$ satisfies the equations

$$
\max _{x \in X} f\left(x, y_{0}\right)=f\left(x_{0}, y_{0}\right)=\min _{y \in Y} f\left(x_{0}, y\right) .
$$

Proof. By the equicontinuity of the family $\{f(x, \cdot): x \in X\}$, for any $y$ in $Y$ there exists an open neighborhood $V_{y}$ of $y$ in $Y$ such that $|f(x, v)-f(x, y)|<1 / 2$ for all $v$ in $V_{y}$ and all $x$ in $X$. Then there exists a finite subset $Z$ of $Y$ such that $Y=\bigcup\left\{V_{z}: z \in Z\right\}$ by the compactness of $Y$. On the other hand, we have $\min _{y \in Y} \sup _{x \in X} f(x, y)<+\infty$ by [7, Theorem 4], and hence there exist a number $M$ and $y_{0}$ in $Y$ such that $f\left(x, y_{0}\right)<M$ for all $x$ in $X$. Let $P_{1}=\left\{z \in Z: y_{0} \in V_{z}\right\}$ and

$$
P_{i+1}=\left\{z \in Z: V_{z} \cap \bigcup\left\{V_{w}: w \in P_{i}\right\} \neq \varnothing\right\} \backslash \bigcup\left\{P_{j}: 1 \leq j \leq i\right\} \quad \text { for } i=1,2, \ldots
$$

Then there exists a positive number $n$ such that $P_{1}, \ldots, P_{n}$ are all nonempty and $P_{n+1}, P_{n+2}, \ldots$ are all empty. We have $Z=P_{1} \cup P_{2} \cup \cdots \cup P_{n}$. In fact, if $Z^{\prime}=$ $Z \backslash P_{1} \cup \cdots \cup P_{n}$ is not empty, then the two open sets $\bigcup\left\{V_{z}: z \in P_{1} \cup \cdots \cup P_{n}\right\}$ and $\bigcup\left\{V_{w}: w \in Z^{\prime}\right\}$ cover $Y$ and they have no intersection, which contradicts the connectedness of $Y$. Hence for any $y$ in $Y$ there exists an integer $m$ with $1 \leq m \leq n$ such that $y \in V_{z}$ for some $z$ in $P_{m}$, and by the construction of $P_{1}, \ldots, P_{n}$, there exists a sequence $y_{1}, \ldots, y_{m}$ in $Y$ with $y_{m}=y$ such that $y_{i-1}$ and $y_{i}$ belong to a neighborhood $V_{z}$ for some $z$ in $P_{i}$ for $i=1, \ldots, m$. Hence for any $x$ in $X$ we have

$$
\begin{aligned}
f(x, y)-f\left(x, y_{0}\right) & \leq\left(f\left(x, y_{0}\right)-f\left(x, y_{1}\right)\right)+\cdots+\left(f\left(x, y_{m-1}\right)-f\left(x, y_{m}\right)\right) \\
& \leq m \leq n .
\end{aligned}
$$

Therefore we have

$$
f(x, y) \leq n+f\left(x, y_{0}\right) \leq n+M=M^{\prime}
$$

for all $x$ in $X$ and all $y$ in $Y$.

If we set $g(y)=\sup _{x \in X} f(x, y)$, then $g$ is a real-valued function on $Y$ and continuous. If fact, let $y_{0}$ be a point of $Y$ and $g\left(y_{0}\right)<r$. If we set $\delta=r-g\left(y_{0}\right)>0$, then there exists neighborhood $V$ of $y_{0}$ such that $f(x, v)-f\left(x, y_{0}\right)<\delta / 2$ for all $x$ in $X$ and ail $v$ in $V$. By the definition of $g$, for any $y$ in $Y$ there exists $x_{y}$ in $X$ such that $f\left(x_{y}, y\right)>g(y)-\delta / 2$. Hence for any $v$ in $V$,

$$
g(v)<f\left(x_{v}, v\right)+\delta / 2<f\left(x_{v}, y_{0}\right)+\delta \leq g\left(y_{0}\right)+\delta=r .
$$

Hence $g$ is upper semicontinuous. Since $g$ is also lower semicontinuous, $g$ is continuous.

We define a multi-valued mapping $A$ of $X$ into $Y$ by

$$
A(x)=\left\{y: f(x, y)=\min _{y \in Y} f(x, y)\right\} .
$$

Then the graph $\operatorname{Gr}(A)$ of $A$ is closed by the continuity of $f$ and the upper semicontinuity of $\min _{y \in Y} f(\cdot, y)$. It is easily seen that $A$ is convex-valued, and hence 
$A$ is closed convex-valued and upper semicontinuous. On the other hand, for any positive integer $k$ we define a multi-valued mapping $B_{k}$ of $Y$ into $X$ by

$$
B_{k}(y)=\{x: f(x, y)>g(y)-1 / k\} \text {. }
$$

Then $B_{k}$ is convex-valued and $B_{k}^{-1}(x)$ is open in $Y$ for all $x$ in $X$. Therefore there exist $x_{k}$ in $X$ and $y_{k}$ in $Y$ such that $x_{k} \in B_{k}\left(y_{k}\right)$ and $y_{k} \in A\left(x_{k}\right)$. Then we have the inequalities

$$
M^{\prime} \geq f\left(x_{k}, y\right) \geq f\left(x_{k}, y_{k}\right)>g\left(y_{k}\right)-1 / k
$$

for all $y$ in $Y$. Since the sequence $\left\{g\left(y_{k}\right)-1 / k\right\}$ is bounded by the continuity of $g$, the sequence $\left\{f\left(x_{k}, \cdot\right)\right\}$ is bounded in the Banach space $C(Y)$. Hence from the Arzelà-Ascoli theorem we may assume that the sequence uniformly converges to a function of the type $f\left(x_{0}, \cdot\right)$ for some $x_{0}$ in $X$. Moreover we may assume that the sequence $\left\{y_{k}\right\}$ converges to a point $y_{0}$ of $Y$ by the compactness of $Y$. Then we have $f\left(x_{0}, y_{0}\right)=\min _{y \in Y} f\left(x_{0}, y\right)$. In fact, if the equation does not hold, then there exists a number $c$ such that $f\left(x_{0}, y_{0}\right)>c>\min _{y \in Y} f\left(x_{0}, y\right)=f\left(x_{0}, y_{1}\right)$ with some $y_{1}$ in $Y$. Since the sequence $\left\{f\left(x_{k}, y_{1}\right)\right\}$ converges to $f\left(x_{0}, y_{1}\right)$, we have

$$
c>f\left(x_{k}, y_{1}\right) \geq \min _{y \in Y} f\left(x_{k}, y\right)=f\left(x_{k}, y_{k}\right)
$$

for sufficiently large $k$. Since the sequence $\left\{f\left(x_{k}, y_{k}\right)\right\}$ converges to $f\left(x_{0}, y_{0}\right)$, we have $f\left(x_{0}, y_{0}\right) \leq c$, which is a contradiction. On the other hand, from the inequality $f\left(x_{k}, y_{k}\right)>g\left(y_{k}\right)-1 / k$, we have $f\left(x_{0}, y_{0}\right) \geq g\left(y_{0}\right)$, that is, $f\left(x_{0}, y_{0}\right)=$ $\max _{x \in X} f\left(x, y_{0}\right)$.

\section{REFERENCES}

1. F. E. Browder, The fixed point theory of multi-valued mappings in topological vector spaces, Math. Ann. 177 (1968), 283-301.

2. __ Coincidence theorems, minimax theorems and variational inequalities, Contemporary Math., Vol. 26, Amer. Math. Soc., Providence, R.I., 1984, pp. 67-80.

3. N. Dunford and J. T. Schwartz, Linear operators, Part I, Wiley, New York, 1971.

4. K. Fan, Fixed point and minimax theorems in locally convex topological linear spaces, Proc. Nat. Acad. Sci. U.S.A. 38 (1952), 121-126.

5. __ A generalization of Tychonoff's fixed point theorem, Math. Ann. 142 (1961), 305310 .

6. $\ldots$, Extensions of two fixed point theorems of F. E. Browder, Math. Z. 112 (1969), $234-240$.

7. C. W. Ha, Minimax and fixed point theorems, Math. Ann. 248 (1980), 73-77.

8. S. Kakutani, A generalization of Brouwer's fixed-point theorem, Duke Math. J. 8 (1941), 457-459.

9. J. L. Kelly and I. Namioka, Linear topological spaces, Springer, New York, 1976.

10. S. Simons, Two-function minimax theorems and variational inequalities for functions on compact and noncompact sets, with some comments on fixed-point theorems, Proc. 1983 Amer. Math. Soc. Summer Inst. on Nonlinear Funct. Anal.; Proc. Sympos. Pure Math. (to appear).

11. W. Takahashi, Nonlinear variational inequalities and fixed point theorems, J. Math. Soc. Japan 28 (1976), 168-181.

12. __ Recent results in fixed point theory, Southeast Asian Bull. Math. 4 (1980), 59-85.

\section{FACUlTy OF EDUCATION, TOtTORI UNIVERSity, TOTtORI 680, JAPAN}

Current address: College of Commerce, Nihon University, 5-2-1 Kinuta, Setagaya-ku, Tokyo 157, Japan 